\title{
THE HAWKES PROCESS WITH DIFFERENT EXCITING FUNCTIONS AND ITS ASYMPTOTIC BEHAVIOR
}

\author{
RAÚl FIERRO, ${ }^{*}$ Pontificia Universidad Católica de Valparaíso and Universidad de Valparaíso \\ VÍCTOR LEIVA,** Universidad de Valparaíso and Universidad Adolfo Ibáñez. \\ JESPER MØLLER, ${ }^{* * *}$ Aalborg University
}

\begin{abstract}
The standard Hawkes process is constructed from a homogeneous Poisson process and uses the same exciting function for different generations of offspring. We propose an extension of this process by considering different exciting functions. This consideration may be important in a number of fields; e.g. in seismology, where main shocks produce aftershocks with possibly different intensities. The main results are devoted to the asymptotic behavior of this extension of the Hawkes process. Indeed, a law of large numbers and a central limit theorem are stated. These results allow us to analyze the asymptotic behavior of the process when unpredictable marks are considered.
\end{abstract}

Keywords: Central limit theorem; clustering effect; law of large numbers; unpredictable marks

2010 Mathematics Subject Classification: Primary 60G55

Secondary 60F05

\section{Introduction}

The standard Hawkes process (HP) is a temporal point process having long memory, clustering effect, and the self-exciting property. The standard HP and its extension to a marked point process are of wide interest, partly because of their many important applications and illustrative examples in the theory of non-Markovian point processes constructed by a conditional intensity. The seminal ideas are due to Hawkes [10], [11] and Hawkes and Oakes [12], whereas useful reviews on the topic are provided by Daley and Vere-Jones [5] and Zhu [22]. Its applications include fields such as finance, genetics, neuroscience, and seismology; see, e.g. Carstensen et al. [4], Embrechts et al. [6], Gusto and Schbath [9], Ogata [17], [18], and Pernice et al. [19].

As mentioned, the standard HP is a cluster process, where the starting points of the clusters are called immigrants and appear according to a homogeneous Poisson process on the nonnegative time axis. Each immigrant is the ancestor of a first generation of offspring, each point of a first generation offspring is the ancestor of a second generation point offspring, and so on. Thereby, the cluster for an immigrant is a set of generations of offspring. More precisely, for a given ancestor appearing at a time $s$, the associated offspring point process is Poisson

\footnotetext{
Received 17 December 2013; revision received 6 February 2014.

* Postal address: Pontificia Universidad Católica de Valparaíso, Brasil 2950, Casilla 4059, Valparaíso, Chile. Email address: rfierro@ucv.cl

** Postal address: Universidad de Valparaíso, Gran Bretaña 1111, Casilla 5030, Valparaíso, Chile. Email address: victorleivasanchez@gmail.com

*** Postal address: Aalborg University, Fredrik Bajers Vej 7G, DK-9220 Aalborg Ø, Denmark. Email address: jm@math.aau.dk
} 
with the intensity function $\gamma(t-s)$, which is defined for $t>s$ and is not dependent on immigrant and offspring points generated before time $s$. Thus, the clusters, conditional to the immigrants, are independent. Note that the same exciting function $\gamma$ is used for all offspring processes. This is the crucial difference to the extension proposed in this paper, where we allow different exciting functions for the different generations of offspring. This extension could be relevant, for instance, in seismology, where main shocks generate aftershocks with different possible intensities.

The main objective of this paper is to investigate the asymptotic behavior of our extension of the HP process. Indeed, a law of large numbers and a central limit theorem are established. Furthermore, by making use of these results, a central limit theorem is proved when unpredictable marks are added to the process. In particular, our asymptotic results do not require the complete identification of offspring processes, but only of the integrals of their exciting functions. We also extend a result obtained by Fierro et al. [7]. Recently, functional central limit theorems for linear and nonlinear HPs were obtained in [1] and [21], respectively. However, their results are based on the standard HP, while ours, coming from a more general definition of the HP, cannot be obtained from these papers. Simulation algorithms and statistical methodology for the extension proposed in this paper remain as open problems to be developed in future studies. For details on exact and approximate simulation algorithms for the standard HP with unpredictable marks, see [14], [15].

This paper is organized as follows. In Section 2, which is divided into four subsections, we introduce our results. In Section 2.1 we define the HP with different exciting functions and establish some preliminary facts. In Section 2.2 we present two of the main results namely, a law of large numbers and a central limit theorem for the process. In Section 2.3 we consider two special cases, one case is the standard HP and the other concerns the case consisting of a finite number of generations. In Section 2.4 we state a central limit theorem for the process with unpredictable marks. The proofs of our results are provided in Section 3.

\section{The Hawkes process with different exciting functions}

\subsection{Definition and preliminary results}

In the sequel, $\left\{\gamma_{n}\right\}_{n \in \mathbb{N}}$ denotes a sequence of locally integrable functions from $\mathbb{R}_{+}$to $\mathbb{R}_{+}$. Here $\mathbb{R}_{+}=[0, \infty)$ is the nonnegative time axis, and $\mathbb{N}=\{0,1, \ldots$,$\} the set of$ nonnegative integers. Given a locally integrable function $\gamma$ from $\mathbb{R}_{+}$to $\mathbb{R}_{+}$, in what follows, by a Poisson process with intensity $\gamma$ we mean a counting process $L$, defined on $\mathbb{R}_{+}$, such that $\left\{L_{t}-\int_{0}^{t} \gamma(u) \mathrm{d} u\right\}_{t \geq 0}$ is a martingale. Note that $L$ could be homogeneous or inhomogeneous, as $\gamma$ is constant or not, respectively.

The following proposition is the basis of what we name the HP with different exciting functions. For concepts related to counting processes and their stochastic intensities; see [2].

Proposition 2.1. There exist a probability space $(\Omega, \mathcal{F}, \mathbb{P})$ and a sequence $\left\{N^{n}\right\}_{n \in \mathbb{N}}$ of nonexplosive counting processes, defined on $\mathbb{R}_{+}$, without common jumps, and satisfying the following three conditions.

(A1) $N^{0}$ is a Poisson process with intensity $\gamma_{0}$.

(A2) For each $n \geq 1, N^{n}$ has predictable stochastic intensity $\lambda^{n}$ given by $\lambda_{t}^{n}=\int_{0}^{t} \gamma_{n}(t-$ s) $\mathrm{d} N_{s}^{n-1}$.

(A3) For each $n \in \mathbb{N}$, conditional to $N^{0}, \ldots, N^{n}, N^{n+1}$ is a Poisson process with intensity $\lambda^{n+1}$. 
Definition 2.1. Let $\left\{N^{n}\right\}_{n \in \mathbb{N}}$ be as in Proposition 2.1 and $N=\sum_{n=0}^{\infty} N^{n}$. We call $N^{0}$ the immigrant process, $N^{n}(n \geq 1)$ the $n$th generation offspring process and $N$ the HP with exciting functions $\left\{\gamma_{n}\right\}_{n \in \mathbb{N}}$.

Remark 2.1. In the standard HP, $\gamma_{0}=\mu$ is constant and $\gamma_{n}=\gamma$ for all $n \geq 1$. In this case there is no need to identify the offspring processes, since $N$ has stochastic intensity $\lambda$ given by $\lambda_{t}=\mu+\int_{0}^{t} \gamma(t-s) \mathrm{d} N_{s}$.

Remark 2.2. In Proposition 2.1, condition (A3) allows us to obtain, recursively, the joint distribution of $N^{0}, \ldots, N^{n}$, for $n \in \mathbb{N}$. It is easy to see that (A2) and (A3) are equivalent.

Remark 2.3. Note that $N$ is univocally defined in distribution. Indeed, according to Theorem 3.6 of [13], there exists, on the Skorohod space, a unique counting process having predictable stochastic intensity $\lambda=\gamma_{0}+\sum_{n=1}^{\infty} \lambda^{n}$.

Let $\Lambda^{n}$ be the compensator of $N^{n}$, that is, for each $n \in \mathbb{N}$ and $t \geq 0, \Lambda_{t}^{n}=\int_{0}^{t} \lambda_{s}^{n} \mathrm{~d} s$, where $\lambda_{s}^{0}=\gamma_{0}(s)$ is a deterministic function. Thus, for each $n \in \mathbb{N}, M^{n}=N^{n}-\Lambda^{n}$ is a $(\mathbb{F}, \mathbb{P})$-martingale, where $\mathbb{F}=\left\{\mathcal{F}_{t}\right\}_{t \geq 0}$ with $\mathcal{F}_{t}=\sigma\left(N_{s}^{0} ; s \leq t\right)$ being the $\sigma$-algebra generated by $\left\{N_{s}^{0} ; 0 \leq s \leq t\right\}$.

Proposition 2.2. For each $n \in \mathbb{N} \backslash\{0\}$ and $t \geq 0, \Lambda_{t}^{n}=\int_{0}^{t} \gamma_{n}(t-s) N_{s}^{n-1} \mathrm{~d} s$.

For two locally integrable functions $f$ and $g$ from $\mathbb{R}_{+}$to $\mathbb{R}, f * g$ denotes the convolution between $f$ and $g$, i.e. $(f * g)(t)=\int_{0}^{t} f(t-s) g(s) \mathrm{d} s$ for $t \geq 0$.

Proposition 2.3. For each $t \geq 0$,

$$
\mathbb{E}\left(N_{t}\right)=\int_{0}^{t} \sum_{n=0}^{\infty}\left(\gamma_{0} * \cdots * \gamma_{n}\right)(u) \mathrm{d} u .
$$

Proposition 2.3 motivates us to consider the following condition:

(B) for each $t \geq 0$, the sequence $\left\{\gamma_{n}\right\}_{n \in \mathbb{N}}$ satisfies

$$
\int_{0}^{t} \sum_{n=0}^{\infty}\left(\gamma_{0} * \cdots * \gamma_{n}\right)(u) \mathrm{d} u<\infty .
$$

Let $M=\sum_{n=0}^{\infty} M^{n}$. Then the HP $N$ is a counting process with compensator $\Lambda=\sum_{n=0}^{\infty} \Lambda^{n}$ and, under condition (B), $M=N-\Lambda$ is a $(\mathbb{F}, \mathbb{P})$-martingale.

For any measurable function $h:[0, \infty) \rightarrow[0, \infty]$, we denote its Laplace transform by $\mathcal{L}[h]$, i.e. for $s \in \mathbb{R}, \mathcal{L}[h](s)=\int_{0}^{\infty} \mathrm{e}^{-s u} h(u) \mathrm{d} u$.

Remark 2.4. Under condition (B), $N$ is a nonexplosive counting process with predictable compensator $\Lambda$.

Proposition 2.4. Condition (B) is satisfied when one of the following conditions holds.

(C1) there exists $s_{0}>0$ such that $\sup _{n \in \mathbb{N}} \mathcal{L}\left[\gamma_{n}\right]\left(s_{0}\right)<1$.

(C2) $\lim _{s \rightarrow \infty} \sup _{k \in \mathbb{N}} \mathcal{L}\left[\gamma_{k}\right](s)=0$.

(C3) There exist $C>0$ and $a>0$ such that $\sup _{k \in \mathbb{N}} \gamma_{k}(t) \leq C \mathrm{e}^{a t}$.

(C4) $\int_{0}^{\infty} \sup _{k \in \mathbb{N}} \gamma_{k}(s) \mathrm{d} s<\infty$.

(C5) $\sup _{k \in \mathbb{N}} \int_{0}^{\infty} \gamma_{k}(s) \mathrm{d} s<1$. 


\subsection{Asymptotic results}

Let $\rho=\sup _{k \in \mathbb{N}} \int_{0}^{\infty} \gamma_{k}(s) \mathrm{d} s$. In this subsection we assume that the following condition holds:

(D) there exists $\overline{\gamma_{0}}=\lim _{t \rightarrow \infty}(1 / t) \int_{0}^{t} \gamma_{0}(s) \mathrm{d} s$ and $\rho<1$.

In particular, from Proposition 2.4, condition (B) holds when condition (D) is satisfied.

In the sequel, $m_{0}=\overline{\gamma_{0}}$, for each $p \in \mathbb{N} \backslash\{0\}, m_{p}=\overline{\gamma_{0}} \prod_{i=1}^{p} \int_{0}^{\infty} \gamma_{i}(u) \mathrm{d} u$, and $m=\sum_{p=0}^{\infty} m_{p}$. Note that, under condition (D), $m<\infty$.

For the standard HP, the condition that $\rho<1$ is usually assumed in order to obtain a nonexplosive process (see, e.g. [5]).

We have the following law of large numbers.

Theorem 2.1. As $t \rightarrow \infty,\left\{N_{t} / t\right\}_{t>0}$ and $\left\{\Lambda_{t} / t\right\}_{t>0}$ converge $\mathbb{P}$-almost surely (a.s.) to $m$, and $\left\{M_{t} / t\right\}_{t>0}$ converges in quadratic mean to 0 .

The following central limit theorem is the main result of this paper. Since additional assumptions on the exciting functions are needed, before we present it, we define $h=\sum_{p=1}^{\infty} h_{p}$, where $h_{p}=\gamma_{p} * \cdots * \gamma_{1}$ for $p \in \mathbb{N} \backslash\{0\}$.

Theorem 2.2. For each $t>0$, let $X_{t}=\left(N_{t}-m t\right) / \sqrt{t}$ and

$$
\sigma_{N}^{2}=\sum_{j=0}^{\infty}\left(1+\sum_{p=1}^{\infty} \prod_{i=j+1}^{p+j} \int_{0}^{\infty} \gamma_{i}(u) \mathrm{d} u\right)^{2} m_{j} .
$$

Additional to condition (D), suppose that the following two conditions hold.

(E1) $\lim _{t \rightarrow \infty} \sqrt{t}\left((1 / t) \int_{0}^{t} \gamma_{0}(u) \mathrm{d} u-\overline{\gamma_{0}}\right)=0$.

(E2) $\lim _{t \rightarrow \infty} \sqrt{t} \int_{t}^{\infty} h(s) \mathrm{d} s=0$.

Then, $\sigma_{N}^{2}<\infty$ and, as $t \rightarrow \infty,\left\{X_{t}\right\}_{t>0}$ converges in distribution to a normal random variable with mean 0 and variance $\sigma_{N}^{2}$.

Remark 2.5. In Theorem 2.2, condition (E1) is satisfied when $\gamma_{0}$ is constant and a sufficient condition for (E2) is:

(E3) $\lim _{t \rightarrow \infty} \int_{t}^{\infty} s^{1 / 2} h(s) \mathrm{d} s=0$.

Proposition 2.5 below gives a simple condition in order for (E3) to hold, which is similar to that considered by Bacry et al. [1].

Proposition 2.5. Let $\alpha \in[0,1)$ and suppose that $S=\sup _{k \in \mathbb{N}} \int_{0}^{\infty} u^{\alpha} \gamma_{k}(u) \mathrm{d} u<\infty$. Then, $\int_{0}^{\infty} u^{\alpha} h(u) \mathrm{d} u<\infty$.

The proofs of Theorems 2.1 and 2.2, provided in Section 3, involve the following three lemmas.

Lemma 2.1. Let $g$ be a nonnegative measurable function defined on $\mathbb{R}_{+}$. Then, for each $t \geq 0$,

$$
\int_{0}^{t}\left(g * \gamma_{0}\right)(v) \mathrm{d} v \leq\left(\int_{0}^{\infty} g(r) \mathrm{d} r\right)\left(\int_{0}^{t} \gamma_{0}(u) \mathrm{d} u\right) .
$$


Lemma 2.2. For each $q \in(0,2]$ there exists $C>0$ such that

$$
\sum_{j=0}^{\infty} \sup _{t>0} \mathbb{E}\left(\sup _{0 \leq u \leq t}\left|M_{u}^{j} / \sqrt{t}\right|^{q}\right) \leq C .
$$

Lemma 2.3. For each integer $p \geq 1$,

$$
\Lambda^{p}=\sum_{j=0}^{p-1} \gamma_{p} * \cdots * \gamma_{j+1} * M^{j}+\gamma_{p} * \cdots * \gamma_{1} * \gamma_{0} * 1
$$

and

$$
\Lambda=\sum_{p=1}^{\infty} \sum_{j=0}^{p-1} \gamma_{p} * \cdots * \gamma_{j+1} * M^{j}+\sum_{p=0}^{\infty} \gamma_{p} * \cdots * \gamma_{1} * \gamma_{0} * 1
$$

Moreover,

$$
\lim _{t \rightarrow \infty} \frac{1}{t} \sum_{p=1}^{\infty} \sum_{j=0}^{p-1} \mathbb{E}\left[\left(\gamma_{p} * \cdots * \gamma_{j+1} *\left|M^{j}\right|\right)_{t}\right]=0
$$

and

$$
\lim _{t \rightarrow \infty} \mathbb{E}\left(\sup _{p \in \mathbb{N}}\left|\frac{\Lambda_{t}^{p}}{t}-m_{p}\right|\right)=0 .
$$

Additional to the above three lemmas, we use the following lemma to prove Theorem 2.2.

Lemma 2.4. For each $j \in \mathbb{N}$ and $u \geq 0, \lim _{t \rightarrow \infty} \mathbb{E}\left(\left|M_{t}^{j}-M_{t-u}^{j}\right|^{2} / t\right)=0$.

\subsection{Two particular cases}

Below we consider two special cases where condition (D) is satisfied and, consequently, the process $\left\{X_{t}\right\}_{t>0}$, defined in Theorem 2.2, has asymptotic normality. Following this we will derive two corollaries of Theorem 2.2.

In the first case, the functions $\gamma_{n}(n \in \mathbb{N} \backslash\{0\})$ are assumed to be equal and, hence, the following result covers the case of the standard HP.

Corollary 2.1. Suppose that the exciting functions $\gamma_{n}=\gamma$ do not depend on $n$, for $n \geq 1$, condition (E1) holds, $\int_{0}^{\infty} \gamma(u) \mathrm{d} u<1$, and $\int_{0}^{\infty} u^{1 / 2} \gamma(u) \mathrm{d} u<\infty$. Then, as $t \rightarrow \infty,\left\{X_{t}\right\}_{t>0}$ converges in distribution to a normal random variable with mean 0 and variance

$$
\sigma_{N}^{2}=\frac{\overline{\gamma_{0}}}{\left(1-\int_{0}^{\infty} \gamma(u) \mathrm{d} u\right)^{3}}
$$

The second particular case is when there exists $n^{*} \in \mathbb{N}$ such that $\gamma_{n^{*}+1}=0$, almost everywhere (a.e.) with respect to the Lebesgue measure. Then, there is at most $n^{*}$ generations of offspring processes. The particular case of $n^{*}=1$ corresponds to a Neyman-Scott cluster point process where the 'mother point process' (i.e. the immigrant process) is included (see, e.g. [16]). 
Corollary 2.2. Suppose that condition (E1) holds and that there exists $n^{*} \in \mathbb{N}$ such that $\gamma_{n^{*}+1}=0$, a.e. with respect to the Lebesgue measure. In addition, we assume that for each $i \in\left\{1, \ldots, n^{*}\right\}, \int_{0}^{\infty} \gamma_{i}(u) \mathrm{d} u<1$ and $\int_{0}^{\infty} u^{1 / 2} \gamma_{i}(u) \mathrm{d} u<\infty$. Then, as $t \rightarrow \infty,\left\{X_{t}\right\}_{t>0}$ converges in distribution to a normal random variable with mean 0 and variance

$$
\sigma_{N}^{2}=\sum_{j=0}^{n^{*}}\left(1+\sum_{p=1}^{n^{*}-j} \prod_{i=j+1}^{p+j} \int_{0}^{\infty} \gamma_{i}(u) \mathrm{d} u\right)^{2} m_{j}
$$

Remark 2.6. It is worth mentioning that an independent proof of Corollary 2.2 could be given with weaker assumptions. Indeed, if for some $n^{*} \in \mathbb{N}, \gamma_{n^{*}+1}=0$, a.e., then in order to obtain the asymptotic normality of $\left\{X_{t}\right\}_{t>0}$, it is enough to assume that $\int_{0}^{\infty} \gamma_{i}(u) \mathrm{d} u<\infty$, for $i \in\left\{1, \ldots, n^{*}\right\}$.

\subsection{Unpredictable marks}

Consider the extension of the standard HP with unpredictable marks defined in [3], [5], and [14] to the case of our HP with different exciting functions, i.e. for each $k \in \mathbb{N}$, we associate a random mark $\xi_{k}$ to the $k$ th jump time $T_{k}$, where these marks are independent identically distributed (i.i.d.) and independent of $N$. (The reader should note that although the terminology 'unpredictable marks' is not used in [3], the meaning of this terminology is simply that the marks are i.i.d. and independent of $N$.) Moreover, assume that the marks are real-valued random variables with mean $v$ and variance $\sigma^{2}$. Under these assumptions, we study the asymptotic distribution of the process $\left\{R_{t}\right\}_{t>0}$ defined by

$$
R_{t}=\frac{1}{\sqrt{t}}\left(\sum_{k=0}^{N_{t}} \xi_{k}-v \mathbb{E}\left(N_{t}\right)\right)
$$

Using the notation of Theorem 2.2, we have the following central limit theorem, which extends a result obtained by Fierro et al. [7].

Theorem 2.3. If conditions (D), (E1), and (E2) are satisfied, then $\left\{R_{t}\right\}_{t>0}$ converges in distribution to a normal random variable with mean 0 and variance $m \sigma^{2}+v^{2} \sigma_{N}^{2}$.

The proof of Theorem 2.3 uses the following lemma.

Lemma 2.5. Let $\left\{U_{t}\right\}_{t>0}$ and $\left\{V_{t}\right\}_{t>0}$ be two real stochastic processes defined on $(\Omega, \mathcal{F}, \mathbb{P})$ and $(U, V)$ be a bivariate random vector defined on the same probability space. Moreover, suppose that the following two conditions hold.

(F1) For any $\varepsilon>0$, there exists $C_{\varepsilon}>0$ such that $\sup _{t>0} \mathbb{P}\left(\max \left\{\left|U_{t}\right|,\left|V_{t}\right|\right\}>C_{\varepsilon}\right)<\varepsilon$.

(F2) For any bounded functions $u$ and $v$ from $\mathbb{R}$ to $\mathbb{R}, \lim _{t \rightarrow \infty} \mathbb{E}\left(u\left(U_{t}\right) v\left(V_{t}\right)\right)=\mathbb{E}(u(U) v(V))$.

Then, as $t \rightarrow \infty,\left\{\left(U_{t}, V_{t}\right)\right\}_{t>0}$ converges in distribution to $(U, V)$.

\section{Proofs}

Below $\mathbf{1}_{A}$ stands for the indicator function of a set $A$.

Proof of Proposition 2.1. Let $(\Omega, \mathcal{F}, \mathbb{P})$ be a complete probability space where a Poisson process $N^{0}$, with intensity $\gamma_{0}$, is defined. Let $\left\{\Lambda_{t}^{1}\right\}_{t \geq 0}$ be the increasing and $(\mathbb{F}, \mathbb{P})$-adapted 
process defined as

$$
\Lambda_{t}^{1}=\int_{0}^{t}\left(\int_{0}^{u} \gamma_{1}(u-s) \mathrm{d} N_{s}^{0}\right) \mathrm{d} u .
$$

Since $\Lambda^{1}$ is predictable and continuous, it follows from Theorem 3.6 of [13] that there exists a counting process $N^{1}$ adapted to the filtration $\mathbb{F}$ with compensator $\Lambda^{1}$. Consequently, for any predictable process $\left\{C_{s}\right\}_{s \geq 0}$, we have

$$
\mathbb{E}\left(\int_{0}^{\infty} C_{s} \mathrm{~d} N_{s}^{1}\right)=\mathbb{E}\left(\int_{0}^{\infty} C_{s} \mathrm{~d} \Lambda_{s}^{1}\right)=\mathbb{E}\left(\int_{0}^{\infty} C_{s} \lambda_{s}^{1} \mathrm{~d} s\right),
$$

where $\lambda_{u}^{1}=\int_{0}^{u} \gamma_{1}(u-s) \mathrm{d} N_{s}^{0}$. This proves that $\lambda^{1}$ is a stochastic intensity for $N^{1}$. Because $N^{0}$ is nonexplosive, for each $t \geq 0, \Lambda_{t}^{1}<\infty, \mathbb{P}$-a.s., which implies that $N^{1}$ is nonexplosive.

Next, suppose that $N^{1}, \ldots, N^{n}$ are nonexplosive counting processes having stochastic intensities $\lambda^{1}, \ldots, \lambda^{n}$, respectively, given by

$$
\lambda_{t}^{m}=\int_{0}^{t} \gamma_{m}(t-s) \mathrm{d} N^{m-1}, \quad 1 \leq m \leq n,
$$

and let $\left\{\Lambda_{t}^{n+1}\right\}_{t \geq 0}$ be the $(\mathbb{F}, \mathbb{P})$-adapted and increasing process defined as

$$
\Lambda_{t}^{n+1}=\int_{0}^{t}\left(\int_{0}^{u} \gamma_{1}(u-s) \mathrm{d} N_{s}^{n}\right) \mathrm{d} u .
$$

We have that $\Lambda^{n+1}$ is predictable and continuous, and as before, Theorem 3.6 of [13] implies that there exists an $(\mathbb{F}, \mathbb{P})$-adapted counting process $N^{n+1}$ with compensator $\Lambda^{n+1}$. Accordingly, for any predictable process $\left\{C_{s}\right\}_{s \geq 0}$, we have

$$
\mathbb{E}\left(\int_{0}^{\infty} C_{s} \mathrm{~d} N_{s}^{n+1}\right)=\mathbb{E}\left(\int_{0}^{\infty} C_{s} \mathrm{~d} \Lambda_{s}^{n+1}\right)=\mathbb{E}\left(\int_{0}^{\infty} C_{s} \lambda_{s}^{n+1} \mathrm{~d} s\right),
$$

where $\lambda_{u}^{n+1}=\int_{0}^{u} \gamma_{n+1}(u-s) \mathrm{d} N_{s}^{n}$. This proves that $\lambda^{n+1}$ is a stochastic intensity for $N^{n+1}$. Since $N^{n}$ is nonexplosive, for each $t \geq 0, \Lambda_{t}^{n+1}<\infty$, $\mathbb{P}$-a.s., which implies that $N^{n+1}$ is nonexplosive. Hence, by induction, $\left\{N^{n}\right\}_{n \in \mathbb{N}}$ is a sequence of nonexplosive counting processes satisfying (A1) and (A2).

Let $n, p \in \mathbb{N}$ with $p>0$. Since $\lambda^{n+p}$ depends on $\omega \in \Omega$ only through $N^{n+p-1}(\omega)$, conditional to $N^{0}, \ldots, N^{n+p-1}, N^{n+p}$ is distributed as a Poisson process with intensity $\lambda^{n+p}$. In particular, (A3) holds. Let us prove that $N^{n}$ and $N^{n+p}$ have no common jumps. Suppose that $T$ is a stopping time such that $\Delta N_{T}^{n}=1, \mathbb{P}$-a.s. Hence, $T$ is measurable with respect to the $\sigma$-algebra generated by $N^{n}$ and, thus,

$$
\begin{aligned}
\mathbb{E}\left(\Delta N_{T}^{n+p} \mid N^{n+p-1}\right) & =\mathbb{E}\left(\int_{0}^{\infty} \mathbf{1}_{\{T\}}(u) \mathrm{d} N_{u}^{n+p} \mid N^{n+p-1}\right) \\
& =\mathbb{E}\left(\int_{0}^{\infty} \mathbf{1}_{\{T\}}(u) \lambda_{u}^{n+p} \mathrm{~d} u \mid N^{n+p-1}\right) \\
& =\int_{0}^{\infty} \mathbf{1}_{\{T\}}(u) \mathbb{E}\left(\lambda_{u}^{n+p} \mid N^{n+p-1}\right) \mathrm{d} u \\
& =0
\end{aligned}
$$


because for each $\omega \in \Omega$, the Lebesgue measure of $\{T(\omega)\}$ equals 0 . Consequently,

$$
\mathbb{E}\left(\Delta N_{T}^{n} \Delta N_{T}^{n+p}\right)=\mathbb{E}\left(\Delta N_{T}^{n} \mathbb{E}\left(\Delta N_{T}^{n+p} \mid N_{T}^{n+p-1}\right)\right)=0,
$$

and, therefore, $\Delta N_{T}^{n} \Delta N_{T}^{n+p}=0, \mathbb{P}$-a.s., which completes the proof.

Proof of Proposition 2.2. By the Fubini theorem and a change of variable, we have

$$
\begin{aligned}
\Lambda_{t}^{n} & =\int_{0}^{t}\left(\int_{0}^{u} \gamma_{n}(u-s) \mathrm{d} N_{s}^{n-1}\right) \mathrm{d} u \\
& =\int_{0}^{t}\left(\int_{0}^{t-s} \gamma_{n}(u) \mathrm{d} u\right) \mathrm{d} N_{s}^{n-1} \\
& =\int_{0}^{t} F_{n}(t-s) \mathrm{d} N_{s}^{n-1},
\end{aligned}
$$

where $F_{n}(t)=\int_{0}^{t} \gamma_{n}(u) \mathrm{d} u$. Integrating by parts, we obtain

$$
\int_{0}^{t} F_{n}(t-s) \mathrm{d} N_{s}^{n-1}=F_{n}(0) N_{t}^{n-1}-F_{n}(t) N_{0}^{n-1}+\int_{0}^{t} \gamma_{n}(t-s) N_{s}^{n-1} \mathrm{~d} s
$$

and, hence, $\Lambda_{t}^{n}=\int_{0}^{t} \gamma_{n}(t-s) N_{s}^{n-1} \mathrm{~d} s$, which concludes the proof.

Proof of Proposition 2.3. Let $\mu_{0}=\gamma_{0}$ and, for each $n \geq 1$ and $t \geq 0, \mu_{n}(t)=\mathbb{E}\left(\lambda_{t}^{n}\right)$. From Proposition 2.2, we have

$$
\mu_{n}(t)=\mathbb{E}\left(\int_{0}^{t} \gamma_{n}(t-s) \mathrm{d} N_{s}^{n-1}\right)=\int_{0}^{t} \gamma_{n}(t-s) \mathbb{E}\left(\lambda_{s}^{n-1}\right) \mathrm{d} s=\left(\gamma_{n} * \mu_{n-1}\right)(t) .
$$

It follows by induction that $\mu_{n}=\gamma_{0} * \gamma_{1} * \cdots * \gamma_{n}$ and, hence,

$$
\sum_{n=0}^{\infty} \mathbb{E}\left(N_{t}^{n}\right)=\int_{0}^{t} \sum_{n=0}^{\infty}\left(\gamma_{0} * \cdots * \gamma_{n}\right)(u) \mathrm{d} u
$$

which concludes the proof.

Proof of Proposition 2.4. Let $H(t)=\mathbb{E}\left(N_{t}\right), r=\sup _{n \in \mathbb{N}} \mathcal{L}\left[\gamma_{n}\right]\left(s_{0}\right)$ and suppose that $(\mathrm{C} 1)$ holds. By Proposition 2.3,

$$
\mathcal{L}[H]\left(s_{0}\right) \leq \frac{1}{s_{0}} \sum_{n=0}^{\infty} r^{n+1}=\frac{r}{s_{0}(1-r)}<\infty .
$$

Consequently, $H<\infty$ a.e. with respect to the Lebesgue measure, and since $H$ is continuous, for each $t \geq 0, H(t)<\infty$, which implies (B).

Note that (C2) implies that there exists $s_{0}>0$ such that $\sup _{k \in \mathbb{N}} \mathcal{L}\left[\gamma_{k}\right]\left(s_{0}\right)<1$. Hence, (C2) implies that (C1) and, consequently, (B) are satisfied. Under (C3), we have

$$
0 \leq \sup _{k \in \mathbb{N}} \mathcal{L}\left[\gamma_{k}\right](s) \leq C \int_{0}^{\infty} \mathrm{e}^{-(s-a) u} \mathrm{~d} u=\frac{C}{s-a},
$$

whenever $s>a$, and, thus, (C3) implies (C2) and, consequently, also (B). 
By the dominated convergence theorem (DCT), (C4) implies (C2) and, hence, (B) holds. Finally,

$$
\int_{0}^{\infty}\left(\gamma_{0} * \cdots * \gamma_{n}\right)(u) \mathrm{d} u=\left(\int_{0}^{\infty} \gamma_{0}(u) \mathrm{d} u\right) \cdots\left(\int_{0}^{\infty} \gamma_{n}(u) \mathrm{d} u\right) \leq\left(\sup _{k \in \mathbb{N}} \int_{0}^{\infty} \gamma_{k}(s) \mathrm{d} s\right)^{n+1}
$$

and, therefore, (C5) implies (B), concluding the proof.

Proof of Proposition 2.5. This proof is similar to that given in Lemma 5 of [1]. Let $A_{p}=$ $\int_{0}^{\infty} t^{\alpha} h_{p}(t) \mathrm{d} t$. We have

$$
\begin{aligned}
A_{p}= & \int_{0}^{\infty} t^{\alpha}\left(\int_{0}^{t} \gamma_{p}(t-u) h_{p-1}(u) \mathrm{d} u\right) \mathrm{d} t \\
= & \int_{0}^{\infty} h_{p-1}(u)\left(\int_{0}^{\infty}(u+s)^{\alpha} \gamma_{p}(s) \mathrm{d} s\right) \mathrm{d} u \\
\leq & \left(\int_{0}^{\infty} h_{p-1}(u) \mathrm{d} u\right)\left(\int_{0}^{\infty} s^{\alpha} \gamma_{p}(s) \mathrm{d} s\right) \mathrm{d} u \\
& +\left(\int_{0}^{\infty} \gamma_{p}(s) \mathrm{d} s\right)\left(\int_{0}^{\infty} u^{\alpha} h_{p-1}(u) \mathrm{d} u\right) \mathrm{d} u \\
\leq & S \rho^{p-1}+\rho A_{p-1} .
\end{aligned}
$$

The case $\rho=0$ is clear and by assuming $\rho>0$, it follows that

$$
\frac{A_{p}}{\rho^{p}}-\frac{A_{p-1}}{\rho^{p-1}} \leq \frac{S}{\rho}
$$

Summing from $p=2$ to $n$ and taking into account that $A_{1} \leq S$, we obtain $A_{n} \leq S n \rho^{n-1}$. Consequently, we have

$$
\int_{0}^{\infty} t^{\alpha} h(t) \mathrm{d} t \leq \frac{S}{(1-\rho)^{2}}<\infty
$$

which concludes the proof.

Proof of Lemma 2.1. It holds that

$$
\begin{aligned}
\int_{0}^{t}\left(g * \gamma_{0}\right)(v) \mathrm{d} v & =\int_{0}^{t}\left(\int_{0}^{v} g(v-u) \gamma_{0}(u) \mathrm{d} u\right) \mathrm{d} v \\
& =\int_{0}^{t} \gamma_{0}(u)\left(\int_{0}^{t-u} g(r) \mathrm{d} r\right) \mathrm{d} u \\
& \leq\left(\int_{0}^{\infty} g(r) \mathrm{d} r\right)\left(\int_{0}^{t} \gamma_{0}(u) \mathrm{d} u\right)
\end{aligned}
$$

which concludes the proof.

Proof of Lemma 2.2. Since $\mathbb{E}\left(\lambda_{t}^{j}\right)=\gamma_{j} * \cdots * \gamma_{1} * \gamma_{0}$, for $t \geq 0$, from Lemma 2.1, we have

$$
\mathbb{E}\left(\Lambda_{t}^{j}\right)=\int_{0}^{t}\left(\gamma_{j} * \cdots * \gamma_{1} * \gamma_{0}\right)(u) \mathrm{d} u \leq \rho^{j} \int_{0}^{t} \gamma_{0}(u) \mathrm{d} u
$$


Hence, the Jensen and Doob inequalities imply that

$$
\mathbb{E}\left(\sup _{0 \leq u \leq t}\left|M_{u}^{j}\right|^{q}\right) \leq \mathbb{E}\left(\sup _{0 \leq u \leq t}\left|M_{u}^{j}\right|^{2}\right)^{q / 2} \leq 2^{q} \mathbb{E}\left(\Lambda_{t}^{j}\right)^{q / 2} \leq 2^{q} \rho^{j q / 2}\left(\int_{0}^{t} \gamma_{0}(u) \mathrm{d} u\right)^{q / 2} .
$$

Thus,

$$
\sup _{t>0} \mathbb{E}\left(\sup _{0 \leq u \leq t}\left|M_{u}^{j} / \sqrt{t}\right|^{q}\right) \leq 2^{q} \rho^{j q / 2} \sup _{t>0}\left(\frac{1}{t} \int_{0}^{t} \gamma_{0}(u) \mathrm{d} u\right)^{q / 2}
$$

and, consequently,

$$
\sum_{j=0}^{\infty} \sup _{t>0} \mathbb{E}\left(\sup _{0 \leq u \leq t}\left|M_{u}^{j} / \sqrt{t}\right|^{q}\right) \leq C
$$

where $C=2^{q} \sup _{t>0}\left((1 / t) \int_{0}^{t} \gamma_{0}(u) \mathrm{d} u\right)^{q / 2} /\left(1-\rho^{q / 2}\right)$. This completes the proof.

Proof of Lemma 2.3. For each $p \in \mathbb{N}, N^{p}=M^{p}+\Lambda^{p}$, and for each $p \geq 1, \Lambda^{p}=\gamma * N^{p-1}$. Hence, (2.1) follows by induction and (2.2) is obtained from (2.1).

Let $F(t)=(1 / t) \sum_{p=1}^{\infty} \sum_{j=0}^{p-1}\left(\gamma_{p} * \cdots * \gamma_{j+1} *\left|M^{j}\right|\right)_{t}$ for $t>0$. Then

$$
\begin{aligned}
|F(t)| & =\left|\frac{1}{t} \sum_{j=0}^{\infty}\left(\left|M^{j}\right| * \sum_{p=j+1}^{\infty} \gamma_{p} * \cdots * \gamma_{j+1}\right)_{t}\right| \\
& \leq \frac{1}{t} \sum_{j=0}^{\infty} \sup _{0 \leq u \leq t}\left|M_{u}^{j}\right| \int_{0}^{\infty} \sum_{p=j+1}^{\infty}\left(\gamma_{p} * \cdots * \gamma_{j+1}\right)(u) \mathrm{d} u \\
& =\frac{1}{t} \sum_{j=0}^{\infty} \sup _{0 \leq u \leq t}\left|M_{u}^{j}\right| \sum_{p=j+1}^{\infty} \prod_{i=j+1}^{p} \int_{0}^{\infty} \gamma_{i}(u) \mathrm{d} u \\
& \leq \frac{\rho}{1-\rho} \sum_{j=0}^{\infty} \sup _{0 \leq u \leq t} \frac{\left|M_{u}^{j}\right|}{t}
\end{aligned}
$$

and from Lemma 2.2, it follows that $\lim _{t \rightarrow \infty} \mathbb{E}(|F(t)|)=0$, which proves (2.3).

Let $h_{p}=\gamma_{p} * \cdots * \gamma_{1}$ and $h=\sum_{p=1}^{\infty} h_{p}$. We have

$$
\begin{aligned}
\frac{1}{t}\left(\gamma_{p} * \cdots * \gamma_{1} * \gamma_{0} * 1\right)(t)= & \frac{1}{t} \int_{0}^{t}\left(h_{p} * \gamma_{0}\right)(u) \mathrm{d} u \\
= & -\int_{0}^{t} h_{p}(s)\left(\frac{1}{t} \int_{t-s}^{t} \gamma_{0}(u) \mathrm{d} u\right) \mathrm{d} s \\
& +\frac{1}{t} \int_{0}^{t} \gamma_{0}(u) \mathrm{d} u \int_{0}^{t} h_{p}(s) \mathrm{d} s
\end{aligned}
$$

and, hence,

$$
\begin{aligned}
\frac{1}{t}\left(\gamma_{p} * \cdots * \gamma_{0} * 1\right)(t)-m_{p}= & {\left[\frac{1}{t} \int_{0}^{t} \gamma_{0}(u) \mathrm{d} u-\overline{\gamma_{0}}\right] \int_{0}^{t} h_{p}(s) \mathrm{d} s } \\
& -\overline{\gamma_{0}} \int_{t}^{\infty} h_{p}(s) \mathrm{d} s \\
& -\int_{0}^{t} h_{p}(s)\left[\frac{1}{t} \int_{t-s}^{t} \gamma_{0}(u) \mathrm{d} u\right] \mathrm{d} s .
\end{aligned}
$$


Thus,

$$
\begin{aligned}
& \sup _{p \in \mathbb{N}}\left|\frac{1}{t}\left(\gamma_{p} * \cdots * \gamma_{1} * \gamma_{0} * 1\right)(t)-m_{p}\right| \\
& \quad \leq\left|\frac{1}{t} \int_{0}^{t} \gamma_{0}(u) \mathrm{d} u-\overline{\gamma_{0}}\right| \int_{0}^{\infty} h(s) \mathrm{d} s+\overline{\gamma_{0}} \int_{t}^{\infty} h(s) \mathrm{d} s+\int_{0}^{\infty} h(s)\left(\frac{1}{t} \int_{t-s}^{t} \gamma_{0}(u) \mathrm{d} u\right) \mathrm{d} s .
\end{aligned}
$$

By making use of $\int_{0}^{\infty} h(s) \mathrm{d} s \leq \rho /(1-\rho)<\infty$ and the DCT, we have

$$
\lim _{t \rightarrow \infty} \sup _{p \in \mathbb{N}}\left|\frac{1}{t}\left(\gamma_{p} * \cdots * \gamma_{1} * \gamma_{0} * 1\right)(t)-m_{p}\right|=0
$$

To prove (2.4) define $A_{t}=\sup _{p \in \mathbb{N}}\left|\left(\Lambda_{t}^{p} / t\right)-m_{p}\right|$ and note that from (2.1) we have

$$
\mathbb{E}\left(A_{t}\right) \leq \mathbb{E}(F(t))+\sup _{p \in \mathbb{N}}\left|\frac{1}{t}\left(\gamma_{p} * \cdots * \gamma_{1} * \gamma_{0} * 1\right)(t)-m_{p}\right| .
$$

From (2.3) and (3.2), we obtain $\lim _{t \rightarrow \infty} \mathbb{E}\left(A_{t}\right)=0$, which proves (2.4) and the proof is complete.

Proof of Theorem 2.1. We have

$$
\mathbb{E}\left(M_{t}^{2}\right)=\sum_{j=0}^{\infty} \mathbb{E}\left(\Lambda_{t}^{j}\right)=\sum_{j=0}^{\infty} \mathbb{E}\left(\left|M_{t}^{j}\right|^{2}\right) .
$$

Hence, from Lemma 2.2 and the DCT, we obtain

$$
\lim _{t \rightarrow \infty} \mathbb{E}\left(\left|M_{t} / t\right|^{2}\right)=\sum_{j=0}^{\infty} \lim _{t \rightarrow \infty} \mathbb{E}\left(\left|\frac{M_{t}^{j}}{t}\right|^{2}\right)=0,
$$

which proves that $\left\{M_{t} / t\right\}_{t>0}$ converges in quadratic mean to 0 .

From (2.2), for each $t>0$, we have

$$
\frac{\Lambda_{t}}{t}=\frac{1}{t} \sum_{p=1}^{\infty} \sum_{j=0}^{p-1} \gamma_{p} * \cdots * \gamma_{j+1} * M^{j}+\frac{1}{t} \sum_{p=1}^{\infty} \gamma_{p} * \cdots * \gamma_{1} * \gamma_{0} * 1 .
$$

Hence, from (2.3) and the Fatôu lemma, in order to prove that $\left\{\Lambda_{t} / t\right\}_{t>0}$ converges $\mathbb{P}$-a.s. to $m$, it suffices to prove that

$$
\lim _{t \rightarrow \infty} \frac{1}{t} \sum_{p=1}^{\infty} \gamma_{p} * \cdots * \gamma_{1} * \gamma_{0} * 1=m
$$

Lemma 2.1 implies that

$$
\begin{aligned}
\sum_{p=1}^{\infty} \sup _{t>0} \frac{1}{t}\left(\gamma_{p} * \cdots * \gamma_{1} * \gamma_{0} * 1\right)(t) & \leq \sup _{t>0}\left(\frac{1}{t} \int_{0}^{t} \gamma_{0}(u) \mathrm{d} u\right) \sum_{p=1}^{\infty} \int_{0}^{\infty}\left(\gamma_{p} * \cdots * \gamma_{1}\right)(r) \mathrm{d} r \\
& \leq \sup _{t>0}\left(\frac{1}{t} \int_{0}^{t} \gamma_{0}(u) \mathrm{d} u\right) \frac{\rho}{1-\rho} \\
& <\infty .
\end{aligned}
$$


Hence, (3.3) follows from the DCT along with (3.2). Since $\left\{M_{t} / t\right\}_{t>0}$ is uniformly integrable, $\left\{M_{t} / t\right\}_{t>0}$ converges $\mathbb{P}$-a.s. to 0 . Thus, $\left\{N_{t} / t\right\}_{t>0}$ converges $\mathbb{P}$-a.s. to $m$ and the proof is complete.

Proof of Lemma 2.4. Let $u \geq 0$. Note that

$$
\lim _{t \rightarrow \infty} \mathbb{E}\left(\frac{\left|M_{t}^{0}-M_{t-u}^{0}\right|^{2}}{t}\right)=\lim _{t \rightarrow \infty} \frac{1}{t} \int_{t-u}^{t} \gamma_{0}(s) \mathrm{d} s=0
$$

and for $j \geq 1$ define $h_{j}=\gamma_{j} * \cdots * \gamma_{1}$, and choose $t^{*}>0$ such that $(1 / t) \int_{0}^{t} \gamma_{0}(v) \mathrm{d} v<\overline{\gamma_{0}}+1$, if $t>t^{*}$.

For $t>t^{*}$ we have

$$
\begin{aligned}
\mathbb{E}\left(\frac{\left|M_{t}^{j}-M_{t-u}^{j}\right|^{2}}{t}\right)= & \mathbb{E}\left[\frac{\Lambda_{t}^{j}-\Lambda_{t-u}^{j}}{t}\right] \\
= & \frac{1}{t} \int_{t-u}^{t}\left(h_{j} * \gamma_{0}\right)(v) \mathrm{d} v \\
= & \int_{0}^{t-u} h_{j}(s)\left(\frac{1}{t} \int_{t-s-u}^{t-s} \gamma_{0}(r) \mathrm{d} r\right) \mathrm{d} s \\
& +\int_{t-u}^{t} h_{j}(s)\left(\frac{1}{t} \int_{0}^{t-s} \gamma_{0}(r) \mathrm{d} r\right) \mathrm{d} s \\
\leq & \int_{0}^{\infty} h_{j}(s)\left(\frac{1}{t} \int_{t-s-u}^{t-s} \gamma_{0}(r) \mathrm{d} r\right) \mathrm{d} s+\left(\overline{\gamma_{0}}+1\right) \int_{t-u}^{t} h_{j}(s) \mathrm{d} s .
\end{aligned}
$$

Since $\int_{0}^{\infty} h_{j}(s) \mathrm{d} s<\infty$, we have $\lim _{t \rightarrow \infty} \int_{t-u}^{t} h_{j}(s) \mathrm{d} s=0$. Moreover, $(1 / t) \int_{t-s-u}^{t-s} \gamma_{0}(r) \mathrm{d} r$ $\leq\left(\overline{\gamma_{0}}+1\right)$ and $\lim _{t \rightarrow \infty}(1 / t) \int_{t-s-u}^{t-s} \gamma_{0}(r) \mathrm{d} r=0$. Hence, the DCT implies that

$$
\lim _{t \rightarrow \infty} \int_{0}^{\infty} h_{j}(s)\left(\frac{1}{t} \int_{t-s-u}^{t-s} \gamma_{0}(r) \mathrm{d} r\right) \mathrm{d} s=0 .
$$

Therefore,

$$
\lim _{t \rightarrow \infty} \mathbb{E}\left(\frac{\left|M_{t}^{j}-M_{t-u}^{j}\right|^{2}}{t}\right)=0
$$

and the proof is complete.

Proof of Theorem 2.2. From (2.2), for each $t>0$,

$$
X_{t}=\frac{1}{\sqrt{t}} M_{t}+\frac{1}{\sqrt{t}} \sum_{p=1}^{\infty} \sum_{j=0}^{p-1} \int_{0}^{t}\left(\gamma_{p} * \cdots * \gamma_{j+1}\right)(u) M_{t-u}^{j} \mathrm{~d} u+D_{0, t},
$$

where

$$
D_{0, t}=\sum_{p=0}^{\infty} \sqrt{t}\left(\frac{1}{t} \int_{0}^{t} \gamma_{p} * \cdots * \gamma_{0}(u) \mathrm{d} u-m_{p}\right)
$$

Let

$$
Y_{t}=\frac{1}{\sqrt{t}} M_{t}+\sum_{p=1}^{\infty} \sum_{j=0}^{p-1} \frac{M_{t}^{j}}{\sqrt{t}} \int_{0}^{\infty}\left(\gamma_{p} * \cdots * \gamma_{j+1}\right)(u) \mathrm{d} u
$$


and $D_{t}=X_{t}-Y_{t}$ for $t>0$. Note that $D_{t}=D_{1, t}-D_{2, t}+D_{0, t}$, where

$$
D_{1, t}=\frac{1}{\sqrt{t}} \sum_{p=1}^{\infty} \sum_{j=0}^{p-1} \int_{0}^{t}\left(\gamma_{p} * \cdots * \gamma_{j+1}\right)(u)\left(M_{t-u}^{j}-M_{t}^{j}\right) \mathrm{d} u
$$

and

$$
D_{2, t}=\sum_{p=1}^{\infty} \sum_{j=0}^{p-1} \frac{M_{t}^{j}}{\sqrt{t}} \int_{t}^{\infty}\left(\gamma_{p} * \cdots * \gamma_{j+1}\right)(u) \mathrm{d} u .
$$

We need to prove that $\left\{D_{0, t}\right\}_{t>0},\left\{D_{1, t}\right\}_{t>0}$ and that $\left\{D_{2, t}\right\}_{t>0}$ converge in probability to 0 .

From (3.1) we have

$$
\begin{aligned}
\left|D_{0, t}\right| \leq & \sqrt{t}\left|\frac{1}{t} \int_{0}^{t} \gamma_{0}(u) \mathrm{d} u-\overline{\gamma_{0}}\right| \int_{0}^{\infty} h(s) \mathrm{d} s+\overline{\gamma_{0}} \sqrt{t} \int_{t}^{\infty} h(s) \mathrm{d} s \\
& +\int_{0}^{\infty} h(s)\left|\frac{1}{\sqrt{t}} \int_{t-s}^{t} \gamma_{0}(u) \mathrm{d} u\right| \mathrm{d} s
\end{aligned}
$$

and condition (E1) implies that

$$
\lim _{t \rightarrow \infty} \sqrt{t}\left(\frac{1}{t} \int_{0}^{t} \gamma_{0}(u) \mathrm{d} u-\overline{\gamma_{0}}\right)=\lim _{t \rightarrow \infty} \frac{1}{\sqrt{t}} \int_{t-s}^{t} \gamma_{0}(u) \mathrm{d} u \mathrm{~d} s=0 .
$$

Since $\int_{0}^{\infty} h(s) \mathrm{d} s \leq \rho /(1-\rho)<\infty$, from the DCT we have

$$
\lim _{t \rightarrow \infty} \int_{0}^{\infty} h(s)\left|\frac{1}{\sqrt{t}} \int_{t-s}^{t} \gamma_{0}(u) \mathrm{d} u\right| \mathrm{d} s=0
$$

and by condition (E2), $\lim _{t \rightarrow \infty} D_{0, t}=0$.

We have

$$
\mathbb{E}\left(\left|D_{1, t}\right|\right) \leq \sum_{p=1}^{\infty} \sum_{j=0}^{p-1} \int_{0}^{\infty}\left(\gamma_{p} * \cdots * \gamma_{j+1}\right)(u) \mathbb{E}\left(\frac{\left|M_{t-u}^{j}-M_{t}^{j}\right|}{\sqrt{t}}\right) \mathrm{d} u
$$

and, since

$$
\frac{\left|M_{t-u}^{j}-M_{t}^{j}\right|}{\sqrt{t}} \leq 2 \frac{\sup _{0 \leq u \leq t}\left|M_{u}^{j}\right|}{\sqrt{t}},
$$

we have $\left(\gamma_{p} * \cdots * \gamma_{j+1}\right)(u) \mathbb{E}\left(\left|M_{t-u}^{j}-M_{t}^{j}\right| / \sqrt{t}\right)$ is bounded by

$$
C_{p, j}(u)=2\left(\gamma_{p} * \cdots * \gamma_{j+1}\right)(u) \sup _{t>0} \mathbb{E}\left(\frac{\sup _{0 \leq u \leq t}\left|M_{u}^{j}\right|}{\sqrt{t}}\right) .
$$

Thus, by Lemma 2.2,

$$
\begin{aligned}
\sum_{p=1}^{\infty} \sum_{j=0}^{p-1} \int_{0}^{\infty} C_{p, j}(u) \mathrm{d} u & =\sum_{j=0}^{\infty} \sum_{p=j+1}^{\infty} \int_{0}^{\infty} C_{p, j}(u) \mathrm{d} u \\
& \leq \frac{2 \rho}{1-\rho} \sum_{j=0}^{\infty} \sup _{t>0} \mathbb{E}\left(\frac{\sup _{0 \leq u \leq t}\left|M_{u}^{j}\right|}{\sqrt{t}}\right) \\
& <\infty
\end{aligned}
$$


Consequently,

$$
\limsup _{t \rightarrow \infty} \mathbb{E}\left(\left|D_{1, t}\right|\right) \leq \sum_{p=1}^{\infty} \sum_{j=0}^{p-1} \int_{0}^{\infty}\left(\gamma_{p} * \cdots * \gamma_{j+1}\right)(u) \limsup _{t \rightarrow \infty} \mathbb{E}\left(\frac{\left|M_{t-u}^{j}-M_{t}^{j}\right|}{\sqrt{t}}\right) \mathrm{d} u .
$$

By the Jensen inequality and Lemma 2.4, $\lim _{\sup } \operatorname{su\infty }_{t \rightarrow \infty} \mathbb{E}\left(\left|M_{t-u}^{j}-M_{t}^{j}\right| / \sqrt{t}\right)=0$, which proves that $\limsup _{t \rightarrow \infty} \mathbb{E}\left(\left|D_{1, t}\right|\right)=0$.

We have

$$
\mathbb{E}\left(\left|D_{2, t}\right|\right) \leq \sum_{p=1}^{\infty} \sum_{j=0}^{p-1} \mathbb{E}\left(\frac{\left|M_{t}^{j}\right|}{\sqrt{t}}\right) \int_{t}^{\infty}\left(\gamma_{p} * \cdots * \gamma_{j+1}\right)(u) \mathrm{d} u
$$

and

$$
\mathbb{E}\left(\frac{\left|M_{t}^{j}\right|}{\sqrt{t}}\right) \sum_{p=j+1}^{\infty} \int_{t}^{\infty}\left(\gamma_{p} * \cdots * \gamma_{j+1}\right)(u) \mathrm{d} u \leq \sup _{t>0} \mathbb{E}\left(\frac{\sup _{0 \leq u \leq t}\left|M_{u}^{j}\right|}{\sqrt{t}}\right) \rho^{p-j} .
$$

Since, by Lemma 2.2,

$$
\sum_{p=1}^{\infty} \sum_{j=0}^{p-1} \sup _{t>0} \mathbb{E}\left(\frac{\sup _{0 \leq u \leq t}\left|M_{u}^{j}\right|}{\sqrt{t}}\right) \rho^{p-j}=\frac{\rho}{1-\rho} \sum_{j=0}^{\infty} \sup _{t>0} \mathbb{E}\left(\frac{\sup _{0 \leq u \leq t}\left|M_{u}^{j}\right|}{\sqrt{t}}\right)<\infty,
$$

we obtain

$$
\lim _{t \rightarrow \infty} \mathbb{E}\left(\left|D_{2, t}\right|\right) \leq \sum_{p=1}^{\infty} \sum_{j=0}^{p-1} \lim _{t \rightarrow \infty} \mathbb{E}\left(\frac{\left|M_{t}^{j}\right|}{\sqrt{t}}\right) \int_{t}^{\infty}\left(\gamma_{p} * \cdots * \gamma_{j+1}\right)(u) \mathrm{d} u .
$$

But $\sup _{t>0} \mathbb{E}\left(\left|M_{t}^{j}\right| / \sqrt{t}\right)<\infty$ and $\int_{0}^{\infty}\left(\gamma_{p} * \cdots * \gamma_{j+1}\right)(u) \mathrm{d} u<\infty$. Consequently,

$$
\lim _{t \rightarrow \infty} \mathbb{E}\left(\left|D_{2, t}\right|\right)=0 \text {. }
$$

Due to the fact that $\left\{D_{1, t}\right\}_{t>0}$ and $\left\{D_{2, t}\right\}_{t>0}$ converge in probability to 0 , it remains only to prove that $\left\{Y_{t}\right\}_{t>0}$ converges in distribution to a normal random variable with mean 0 and variance $\sigma_{N}^{2}$. To this purpose, we use Theorem 1 of [20, Chapter 8]. For each $j \in \mathbb{N}$, let

$$
\alpha_{j}=1+\sum_{p=1}^{\infty} \prod_{i=j+1}^{p+j} \int_{0}^{\infty} \gamma_{i}(u) \mathrm{d} u
$$

and note that $Y_{t}=Z_{t} / \sqrt{t}$, where $Z=\left\{Z_{t}\right\}_{t \geq 0}$ is given by $Z_{t}=\sum_{j=0}^{\infty} \alpha_{j} M_{t}^{j}$. Since $\sup _{j \in \mathbb{N}} \alpha_{j}<\infty$, we have

$$
\mathbb{E}\left(Z_{t}^{2}\right) \leq \sup _{j \in \mathbb{N}} \alpha_{j}^{2} \sum_{j=0}^{\infty} \mathbb{E}\left(\left|M_{t}^{j}\right|^{2}\right)=\sup _{j \in \mathbb{N}} \alpha_{j}^{2} \mathbb{E}\left(N_{t}\right)<\infty .
$$

Moreover, the martingales $M^{j}(j \in \mathbb{N})$ have no common jumps. Hence, the predictable quadratic variation of the martingale $\left\{Z_{t}\right\}_{t \geq 0}$ is given, for each $t \geq 0$, by

$$
\langle Z\rangle_{t}=\sum_{j=0}^{\infty} \alpha_{j}^{2}\left\langle M^{j}\right\rangle_{t}=\sum_{j=0}^{\infty} \alpha_{j}^{2} \Lambda_{t}^{j}
$$


As usual, $[t]$ denotes the integer part of $t(t>0)$. By making use of Lemma 2.2, it is easy to see that $\left\{Y_{t}-Y_{[t]}\right\}_{t>0}$ converges in probability to 0 . Consequently, in order to prove the convergence of $\left\{Y_{t}\right\}_{t>0}$, it suffices to prove $\left\{Y_{n}\right\}_{n \in \mathbb{N} \backslash\{0\}}$ converges in distribution to a normal random variable with mean 0 and variance $\sigma_{N}^{2}$.

For $n \geq 1$, define $\xi_{n, k}=\left(Z_{k}-Z_{k-1}\right) / \sqrt{n}$ for $k=0, \ldots, n$, where $Z_{-1}=0$. Hence, $\left\{\xi_{n, k}\right\}_{0 \leq k \leq n}$ is a martingale-difference array with respect to $\left\{\varepsilon_{n, k}\right\}_{0 \leq k \leq n}$, where for each $n \in \mathbb{N}$, $\varepsilon_{n, k}=\mathscr{F}_{k}$. That is, $\xi_{n, k}$ is $\mathcal{E}_{n, k}$ measurable, for $k=0, \ldots, n$ and $\mathbb{E}\left(\xi_{n, k} \mid \mathcal{E}_{n, k-1}\right)=0$ for $k=1, \ldots, n$.

Note that

$$
\sum_{k=1}^{n} \mathbb{E}\left(\xi_{n, k}^{2} \mid \mathcal{E}_{n, k-1}\right)=\sum_{k=1}^{n} \sum_{j=0}^{\infty} \frac{\alpha_{j}^{2}\left(\Lambda_{k}^{j}-\Lambda_{k-1}^{j}\right)}{n}=\sum_{j=0}^{\infty} \frac{\alpha_{j}^{2} \Lambda_{n}^{j}}{n}
$$

and

$$
\sum_{k=1}^{n} \mathbb{E}\left(\xi_{n, k}^{2} \mid \varepsilon_{n, k-1}\right)-\sigma_{N}^{2}=\sum_{j=0}^{\infty} \alpha_{j}^{2}\left(\frac{\Lambda_{n}^{j}}{n}-m_{j}\right)
$$

Thus,

$$
\mathbb{E}\left|\sum_{k=1}^{n} \mathbb{E}\left(\xi_{n, k}^{2} \mid \mathcal{E}_{n, k-1}\right)-\sigma_{N}^{2}\right| \leq \sum_{j=0}^{\infty} \alpha_{j}^{2} \mathbb{E}\left|\frac{\Lambda_{n}^{j}}{n}-m_{j}\right| .
$$

Note that if $m_{j^{*}}=0$ for some $j^{*} \in \mathbb{N}$, from (2.4) we have

$$
\lim _{n \rightarrow \infty} \sum_{j=0}^{\infty} \alpha_{j}^{2} \mathbb{E}\left|\frac{\Lambda_{n}^{j}}{n}-m_{j}\right|=\lim _{n \rightarrow \infty} \sum_{j=0}^{j^{*}-1} \alpha_{j}^{2} \mathbb{E}\left|\frac{\Lambda_{n}^{j}}{n}-m_{j}\right|=0 .
$$

Next, assume that $m_{j} \neq 0$ for all $j \in \mathbb{N}$. This implies that $\overline{\gamma_{0}} \neq 0$ and, from (2.1) and Lemma 2.1, we obtain

$$
\begin{aligned}
\sup _{n \geq 1, j \in \mathbb{N}} \mathbb{E}\left(\frac{\Lambda_{n}^{j}}{n m_{j}}\right) & \leq \sup _{n \geq 1, j \in \mathbb{N}}\left(\frac{1}{m_{j}} \int_{0}^{\infty}\left(\gamma_{j} * \cdots * \gamma_{1}\right)(u) \mathrm{d} u\right)\left(\frac{1}{n} \int_{0}^{n} \gamma_{0}(u) \mathrm{d} u\right) \\
& =\sup _{n \geq 1, j \in \mathbb{N}}\left(\frac{1}{m_{j}} \prod_{i=1}^{j} \int_{0}^{\infty} \gamma_{i}(u) \mathrm{d} u\right)\left(\frac{1}{n} \int_{0}^{n} \gamma_{0}(u) \mathrm{d} u\right) \\
& =\sup _{n \geq 1} \frac{1}{\overline{\gamma_{0}} n} \int_{0}^{n} \gamma_{0}(u) \mathrm{d} u \\
& <\infty .
\end{aligned}
$$

Since

$$
\mathbb{E}\left|\frac{\Lambda_{n}^{j}}{n}-m_{j}\right| \leq m_{j} \mathbb{E}\left|\frac{\Lambda_{n}^{j}}{m_{j} n}-1\right| \leq(C+1) m_{j},
$$

where $C=\sup _{n \geq 1, j \in \mathbb{N}} \mathbb{E}\left(\Lambda_{n}^{j} / n m_{j}\right)$ and $\sum_{j=0}^{\infty} m_{j}=m<\infty$, from (2.4) in Lemma 2.3 we obtain

$$
\lim _{n \rightarrow \infty} \sum_{j=0}^{\infty} \alpha_{j}^{2} \mathbb{E}\left|\frac{\Lambda_{n}^{j}}{n}-m_{j}\right|=\sum_{j=0}^{\infty} \alpha_{j}^{2} \lim _{n \rightarrow \infty} \mathbb{E}\left|\frac{\Lambda_{n}^{j}}{n}-m_{j}\right|=0 .
$$


Hence,

$$
\lim _{n \rightarrow \infty} \mathbb{E}\left|\sum_{k=1}^{n} \mathbb{E}\left(\xi_{n, k}^{2} \mid \varepsilon_{n, k-1}\right)-\sigma_{N}^{2}\right|=0 .
$$

To complete the proof, we need to verify that $\left\{\xi_{n, k}\right\}_{0 \leq k \leq n}$ satisfies the Lindeberg condition stated in Theorem 1 of [20, Chapter 8]. For this purpose, we prove that the sequence $\left\{\max _{0 \leq k \leq n} \xi_{n, k}\right\}_{n \in \mathbb{N} \backslash\{0\}}$ is uniformly integrable and converges in probability to 0 (see, e.g. [8, pages $314-315])$. To this purpose, it suffices to prove that

$$
\lim _{n \rightarrow \infty} \mathbb{E}\left(\max _{0 \leq k \leq n} \xi_{n, k}^{2}\right)=0 .
$$

Let $k_{n}^{*}=\min \left\{k \leq n: \xi_{n, k}^{2}=\max _{0 \leq k \leq n} \xi_{n, k}^{2}\right.$ or $\left.k=n\right\}$. By the Doob optional sampling theorem, we have

$$
\mathbb{E}\left(\max _{0 \leq k \leq n} \xi_{n, k}^{2}\right)=\mathbb{E}\left(\xi_{n, k_{n}^{*}}^{2}\right)=\sum_{j=0}^{\infty} \alpha_{j}^{2} c_{n}^{j}
$$

where $c_{n}^{j}=\mathbb{E}\left(\Lambda_{k_{n}^{*}}^{j}-\Lambda_{k_{n}^{*}-1}^{j}\right) / n$. Lemma 2.1 and (2.1) imply that

$$
c_{n}^{j}=\frac{1}{n} \mathbb{E}\left(\int_{k_{n}^{*}-1}^{k_{n}^{*}}\left(\gamma_{j} * \cdots * \gamma_{1} * \gamma_{0}\right)(u) \mathrm{d} u\right) \leq 2 \rho^{j} \sup _{t>0} \frac{1}{t} \int_{0}^{t} \gamma_{0}(u) \mathrm{d} u
$$

and $\sup _{t>0}(1 / t) \int_{0}^{t} \gamma_{0}(u) \mathrm{d} u \sum_{j=0}^{\infty} \alpha_{j}^{2} \rho^{j}<\infty$. Consequently, from (3.5), in order to obtain (3.4) it remains only to prove that for each $j \in \mathbb{N}, \lim _{n \rightarrow \infty} c_{n}^{j}=0$.

We have

$$
\begin{aligned}
c_{n}^{j} & \leq \frac{1}{n} \max _{0 \leq k \leq n} \int_{k-1}^{k}\left(\gamma_{j} * \cdots * \gamma_{1} * \gamma_{0}\right)(u) \mathrm{d} u \\
& =\frac{1}{n} \int_{k_{n}-1}^{k_{n}}\left(\gamma_{j} * \cdots * \gamma_{1} * \gamma_{0}\right)(u) \mathrm{d} u \\
& =\frac{1}{n} \mathbb{E}\left(\Lambda_{k_{n}}^{j}-\Lambda_{k_{n}-1}^{j}\right),
\end{aligned}
$$

where $0 \leq k_{n} \leq n$. Hence, from (2.4) we obtain $\lim _{n \rightarrow \infty} c_{n}^{j}=0$. Therefore, (3.4) holds and the proof is complete.

Proof of Lemma 2.5. For each $C>0$, let $\varphi_{C}$ be the function from $\mathbb{R}$ to $\mathbb{R}$ defined as

$$
\varphi_{C}(x)= \begin{cases}-C & \text { if } x<-C, \\ x & \text { if } C \leq x \leq C, \\ C & \text { if } x>C .\end{cases}
$$

Due to (F1), it suffices to prove that, for each $C>0,\left\{\left(\varphi_{C}\left(U_{t}\right), \varphi_{C}\left(V_{t}\right)\right)\right\}_{t>0}$ converges in distribution to $\left(\varphi_{C}(U), \varphi_{C}(V)\right)$. Fix $C>0$ and let $f$ be a bounded and continuous function from $\mathbb{R}^{2}$ to $\mathbb{R}$ and $\varepsilon>0$. From the Stone-Weierstrass theorem, there exist $u_{1}, \ldots, u_{r}$ and $v_{1}, \ldots, v_{r}$, real continuous functions, defined on $K=[-C, C] \times[-C, C]$ such that

$$
\sup _{(x, y) \in K}\left|f(x, y)-\sum_{i=1}^{r} u_{i}(x) v_{i}(y)\right|<\varepsilon .
$$


Hence,

$\left|\mathbb{E}\left[f\left(\varphi_{C}\left(U_{t}\right)\right), \varphi_{C}\left(V_{t}\right)\right]-\mathbb{E}\left[f\left(\varphi_{C}(U)\right), \varphi_{C}(V)\right]\right|$

$$
\leq\left|\sum_{i=1}^{r} \mathbb{E}\left[u_{i}\left(\varphi_{C}\left(U_{t}\right)\right) v_{i}\left(\varphi_{C}\left(V_{t}\right)\right)\right]-\mathbb{E}\left[u_{i}\left(\varphi_{C}(U)\right) v_{i}\left(\varphi_{C}(V)\right)\right]\right|+2 \varepsilon
$$

and from (F2), we obtain

$$
\limsup _{t \rightarrow \infty}\left|\mathbb{E}\left[f\left(\varphi_{C}\left(U_{t}\right)\right), \varphi_{C}\left(V_{t}\right)\right]-\mathbb{E}\left[f\left(\varphi_{C}(U)\right), \varphi_{C}(V)\right]\right| \leq 2 \varepsilon .
$$

Since $\varepsilon>0$ is arbitrary, the proof is complete.

Proof of Theorem 2.3. For each $n \in \mathbb{N} \backslash\{0\}$ and $t>0$, let

$$
X_{n}=\frac{1}{\sqrt{n}} \sum_{k=0}^{n}\left(\xi_{k}-v\right) \quad \text { and } \quad Y_{t}=v\left(\frac{N_{t}-\mathbb{E}\left(N_{t}\right)}{\sqrt{t}}\right) .
$$

We have $\left\{X_{n}\right\}_{n \in \mathbb{N} \backslash\{0\}}$ and $\left\{Y_{t}\right\}_{t>0}$ are independent and

$$
R_{t}=\sqrt{\frac{N_{t}}{t}} X_{N_{t}}+Y_{t}
$$

By the standard central limit theorem and Theorem 2.2, $\left\{X_{n}\right\}_{n \in \mathbb{N} \backslash\{0\}}$ and $\left\{Y_{t}\right\}_{t>0}$ converge in distribution to two normal random variables $X$ and $Y$, respectively. We assume that $X$ and $Y$ are defined on $(\Omega, \mathcal{F}, \mathbb{P})$ and, hence, they are independent. By Theorem 2.1, (3.6), and the Slutsky theorem, it suffices to prove that $\left\{\left(X_{N_{t}}, Y_{t}\right)\right\}_{t>0}$ converges in distribution to $(X, Y)$. For this purpose, we use Lemma 2.5. Since $\left\{X_{N_{t}}\right\}_{t>0}$ and $\left\{Y_{t}\right\}_{t>0}$ are convergent in distribution, we have $\left\{\left(X_{N_{t}}, Y_{t}\right)\right\}_{t>0}$ satisfies (F1). Let $u$ and $v$ be continuous and bounded functions from $\mathbb{R}$ to $\mathbb{R}, c_{u}=\sup _{x \in \mathbb{R}}|u(x)|$, and $c_{v}=\sup _{x \in \mathbb{R}}|v(x)|$. Since $\left\{X_{t}\right\}_{t>0}$ converges in distribution to $X$, there exists $t^{*} \geq 0$ such that $\left|\mathbb{E}\left[u\left(X_{t}\right)-u(X)\right]\right|<\varepsilon$, for all $t>t^{*}$.

Since $X$ is independent of $\left\{Y_{t}\right\}_{t>0}$ and $Y$, we have

$$
\begin{aligned}
\left|\mathbb{E}\left(u\left(X_{N_{t}}\right) v\left(Y_{t}\right)-u(X) v(Y)\right)\right| \leq & \left|\mathbb{E}\left(\left[u\left(X_{N_{t}}\right)-u(X)\right] v\left(Y_{t}\right)\right)\right|+\left|\mathbb{E}\left(u(X)\left[v\left(Y_{t}\right)-v(Y)\right]\right)\right| \\
\leq & \left|\mathbb{E}\left[\left(u\left(X_{N_{t}}\right)-u(X)\right) v\left(Y_{t}\right) \mathbf{1}_{\left\{N_{t}>t^{*}\right\}}\right]\right| \\
& +2 c_{u} c_{v} \mathbb{P}\left(N_{t} \leq t^{*}\right)+c_{u}\left|\mathbb{E}\left[v\left(Y_{t}\right)-v(Y)\right]\right| .
\end{aligned}
$$

For each $\omega \in\left\{N_{t}>t^{*}\right\}$, we have

$$
\begin{aligned}
\mid \mathbb{E}[(u & \left.\left.\left(X_{N_{t}}\right)-u(X)\right) v\left(Y_{t}\right) \mathbf{1}_{\left\{N_{t}>t^{*}\right\}} \mid N_{t}\right](\omega) \mid \\
& =\left|v\left(Y_{t}(\omega)\right) \mathbb{E}\left[\left(u\left(X_{N_{t}}\right)-u(X)\right) \mathbf{1}_{\left\{N_{t}>t^{*}\right\}} \mid N_{t}\right](\omega)\right| \\
& \leq c_{v}\left|\mathbb{E}\left[\left(u\left(X_{N_{t}(\omega)}\right)-u(X)\right) \mathbf{1}_{\left\{N_{t}>t^{*}\right\}} \mid N_{t}\right](\omega)\right| \\
& =c_{v}\left|\mathbb{E}\left[\left(u\left(X_{N_{t}(\omega)}\right)-u(X)\right)\right]\right| \mathbf{1}_{\left\{N_{t}>t^{*}\right\}}(\omega) \\
& <c_{v} \varepsilon .
\end{aligned}
$$

Consequently,

$$
\left|\mathbb{E}\left(u\left(X_{N_{t}}\right) v\left(Y_{t}\right)-u(X) v(Y)\right)\right| \leq c_{v} \varepsilon+2 c_{u} c_{v} \mathbb{P}\left(N_{t} \leq t^{*}\right)+c_{u}\left|\mathbb{E}\left[v\left(Y_{t}\right)-v(Y)\right]\right| .
$$

But $\varepsilon>0$ is arbitrary and $\lim _{t \rightarrow \infty}\left\{2 c_{u} c_{v} \mathbb{P}\left(N_{t} \leq t^{*}\right)+c_{u}\left|\mathbb{E}\left[v\left(Y_{t}\right)-v(Y)\right]\right|\right\}=0$. Therefore, $\lim _{t \rightarrow \infty}\left|\mathbb{E}\left[u\left(X_{N_{t}}\right) v\left(Y_{t}\right)-u(X) v(Y)\right]\right|=0$ and, by Lemma 2.5 , the proof is complete. 


\section{Acknowledgements}

We are very grateful to an anonymous referee for his/her careful review and of this paper and for the suggestions which allowed us to improve the content. The research of Raúl Fierro and Víctor Leiva was partially supported by the Chilean Council for Scientific and Technological Research, grant FONDECYT 1120879. The research of Jesper Møller was supported by the Danish Council for Independent Research for Natural Sciences, grant 12-124675, 'Mathematical and Statistical Analysis of Spatial Data', and by the Centre for Stochastic Geometry and Advanced Bioimaging, funded by a grant from the Villum Foundation.

\section{References}

[1] Bacry, E., Delattre, S., Hoffmann, M. and Muzy, J. F. (2013). Some limit theorems for Hawkes processes and application to financial statistics. Stoch. Process. Appl. 123, 2475-2499.

[2] Brémaud, P. (1981). Point Processes and Queues. Martingale Dynamics. Springer, New York.

[3] Brémaud, P. Nappo, G. AND Torrisi, G. L. (2002). Rate of convergence to equilibrium of marked Hawkes processes. J. Appl. Prob. 39, 123-136.

[4] Carstensen, L., Sandelin, A., Winther, O. And Hansen, N. R. (2010). Multivariate Hawkes process models of the occurrence of regulatory elements. BMC Bioinformatics 11, 456.

[5] Daley, D. J. And Vere-Jones, D. (2003). An Introduction to the Theory of Point Processes, Vol. 1, Elementary Theory and Methods, 2nd edn. Springer, New York.

[6] Embrechts, P., Liniger, T. And Lu, L. (2011). Multivariate Hawkes processes: an application to financial data. In New Frontiers in Applied Probability (J. Appl. Prob. Spec. Vol. 48A), Applied Probability Trust, Sheffield, pp. 367-378.

[7] Fierro, R., Leiva, V., Ruggeri, F. and Sanhueza, A. (2013). On a Birnbaum-Saunders distribution arising from a non-homogeneous Poisson process. Statist. Prob. Lett. 83, 1233-1239.

[8] Gänssler, P. and Haeusler, E. (1986). On martingale central limit theory. In Dependence in Probability and Statistics, Birkhäuser, Boston, MA, pp. 303-334.

[9] Gusto, G. And SchватH, S. (2005). FADO: a statistical method to detect favored or avoided distances between occurrences of motifs using the Hawkes' model. Statist. Appl. Genet. Molec. Biol. 4, 24.

[10] Hawkes, A. G. (1971). Point spectra of some mutually exciting point processes. J. R. Statist. Soc. Ser. B 33, 438-443.

[11] Hawkes, A. G. (1971). Spectra of some self-exciting and mutually exciting point processes. Biometrika 58, 83-90.

[12] Hawkes, A. G. and Oakes, D. (1974). A cluster process representation of a self-exciting process. J. Appl. Prob. 11, 493-503.

[13] JACOD, J. (1974). Multivariate point processes: predictable projection, Radon-Nikodým derivatives, representation of martingales. Z. Wahrscheinlichkeitsth. 31, 235-253.

[14] Møller, J. and Rasmussen, J. G. (2005). Perfect simulation of Hawkes processes. J. Appl. Prob. 37, $629-646$.

[15] Møller, J. And Rasmussen, J. G. (2006). Approximate simulation of Hawkes processes. Methodology Comput. Appl. Prob. 8, 53-64.

[16] Møller, J. and WaAgepetersen, R. P. (2004). Statistical Inference and Simulation for Spatial Point Processes. Chapman \& Hall/CRC, Boca Raton, FL.

[17] Ogata, Y. (1988). Statistical models for earthquake occurrences and residual analysis for point processes. J. Amer. Statist. Assoc. 83, 9-27.

[18] Ogata, Y. (1998). Space-time point-process models for earthquake occurrences. Ann. Inst. Statist. Math. 50, 379-402.

[19] Pernice, V., Staude, B., Cardanobile, S. and Rotter, S. (2012). Recurrent interactions in spiking networks with arbitrary topology. Phys. Rev. E 85, 031916.

[20] Pollard, D. (1984). Convergence of Stochastic Processes. Springer, New York.

[21] ZHU, L. (2013). Central limit theorem for nonlinear Hawkes processes. J. Appl. Prob. 50, 760-771.

[22] ZHU, L. (2013). Nonlinear Hawkes processes. Doctoral Thesis, New York University. 